\title{
THE ROLE OF IRON DEFICIENCY IN EXPERIMENTALLY-INDUCED ORAL CANDIDOSIS IN THE RAT
}

\author{
J. S. Rennie, A. W. Hutcheon*, T. W. MacFarlane and D. G. MacDonald \\ Department of Oral Medicine and Pathology, Glasgow Dental Hospital and School, \\ Glasgow G2 $3 J Z$ and *Department of Medicine, Western Infirmary, Glasgow
}

\begin{abstract}
SUMmaRY. In comparison with normal rats, those with iron deficiency anaemia showed no significant difference in susceptibility to experimental infection with Candida albicans although anaemic rats had a significantly greater incidence of persistent infection. These findings support the suggestion that patients with chronic candidosis should be investigated for iron deficiency.
\end{abstract}

\section{INTRODUCTION}

In recent years there has been increasing interest in the role of nutritional factors in host-parasite relationships. Micro-organisms have an absolute requirement for iron and this may be more than the simple requirement for an essential growth factor because, in experimental animals, the protective effect of antisera against specific micro-organisms can be completely abolished by the simultaneous injection of iron (Bullen, Ward and Wallis, 1974; Elin and Wolff, 1974). Clinical evidence also suggests that the availability of iron is important in the establishment of infections. Infection has been reported in diseases accompanied by red cell destruction and elevated serum iron concentrations. Sickle cell anaemia is associated with salmonella osteomyelitis (Ringelhann, Konotey-Ahulu and Dodu, 1970) and malarial patients have a higher than expected incidence of systemic salmonellosis (Black, Kunz and Swartz, 1960). In acute leukaemia, the serum iron concentration is raised and, in many cases, serum transferrin is fully saturated. It has been suggested that these changes are causally related to the high incidence of candidosis and other fungal opportunistic infections occurring in leukaemia (Caroline, Rosner and Kozinn, 1969).

It is also thought that in certain circumstances iron deficiency may contribute to resistance to infection. The increased resistance to infection is said to result from high serum levels of transferrin, a bactericidal and fungistatic protein (Schade and Caroline, 1944 and 1946), and low levels of serum iron. In support of this concept Masawe, Muindi and Swai (1974) reported a decreased susceptibility to infection in iron deficient patients. Yet not all the available evidence supports the view that iron deficiency contributes to resistance to infection. Iron deficiency has been reported to depress the ability of neutrophil polymorphonuclear leukocytes to kill bacteria

Received 31 Dec. 1982; accepted 26 Jan. 1983.

* Present address: Woodend General Hospital, Aberdeen AB9 2YS. 
(Chandra, 1973) and to reduce the lymphocyte response to antigenic stimulation (Joynson et al., 1972; Fletcher et al., 1975).

Several investigators have noticed an association between oral candidosis and iron deficiency. Cawson (1963) described two patients, in a series with sore mouths related to dentures, who had iron deficiency anaemia and in whom candidal infection regressed after replacement therapy alone. Fletcher et al. (1975) noted a high incidence of candidal infection in iron-deficient patients, but Jenkins et al. (1977) failed to show any effect of iron deficiency upon the isolation of Candida albicans from the oral mucous membranes.

Thus there is some doubt about the role of iron deficiency in oral candidosis. Clinically, co-existing vitamin deficiencies, host immunity, drug therapy and local factors such as oral hygiene and prostheses may be important and may explain some of the differences in reported results. Carefully-controlled animal experiments are free from many of these problems and might facilitate a better understanding of any relationship between iron deficiency and oral candidosis.

Russell and Jones (1973) described an animal model for oral candidosis that involved oral administration of $C$. albicans to rats treated with tetracycline hydrochloride. C. albicans is not normally present in the commensal oral flora of the rat and although normal rats can be infected by oral administration alone, the carriage rate is low. Use of tetracycline greatly increases the oral carriage rate of the organism and provides a useful model. The rat has the additional advantage of being useful for the study of iron metabolism, because there are several dietary methods for producing iron deficiency in rats (McCall et al., 1972; Amine and Hegsted, 1971).

The aim of this study was to investigate any association between iron deficiency and experimental oral candidosis in the rat model described by Russell and Jones (1973).

\section{MATERIALS AND METHODS}

Rats. Five groups of young, adult male Sprague Dawley rats were age-matched and divided into an untreated control group of five animals, and four experimental groups as shown in the table.

Anaemia was induced by feeding an iron-free vitamin-supplemented diet (McCall et al., 1962) for 3 months before induction of candidal infection. Ten animals selected at random had blood withdrawn to check the haemoglobin level before administration of $C$. albicans. All animals had haemoglobin values below $11 \mathrm{~g} / \mathrm{dl}$. Animals allocated to receive tetracycline were given a solution of tetracycline hydrochloride $0.1 \%$ in the drinking water for 1 week before the first administration and a $0.01 \%$ solution for the remainder of the experiment (Russell and Jones, 1973).

C. albicans MRL 3153 (obtained from the Mycological Reference Laboratory, London) was used throughout the study. The yeast was cultured for $48 \mathrm{~h}$ at $37^{\circ} \mathrm{C}$ on Sabouraud's agar, and the resulting growth harvested in normal saline and washed once by centrifugation at $2500 \mathrm{rpm}$ for $10 \mathrm{~min}$. The organisms were resuspended in normal saline and a suspension prepared with $10^{7}-10^{8}$ yeasts $/ \mathrm{ml}$. Oral candidal infection was induced by administering $0.2 \mathrm{ml}$ of this suspension to unanaesthetised animals on six occasions. Each animal received two doses each week for 2 weeks and one dose each week for a further 2 weeks. Before administration of $C$. albicans, the mouth of each rat was gently swabbed and the sample inoculated on to Sabouraud's agar. Plates were incubated at $37^{\circ} \mathrm{C}$ for $48 \mathrm{~h}$ and examined for yeast colonies that were confirmed as $C$. albicans by the germ tube test (Mackenzie, 1962) and by sugar fermentation reactions (Lodder, 1970). After 5 weeks (1 week after the last dose) animals were killed by an overdose of intra-peritoneal barbiturate. Because of the larger numbers of animals in the antibiotic treated groups, animals in these groups were killed in three groups during a period of 3 weeks. Oral 
swabs were taken and examined for the presence of candida as previously described. Blood was collected for haematological and biochemical examination. To assess whether or not oral mucosal infestation had occurred, the hard and soft palate, the tongue and the cheek mucosa mucosa were dissected and trimmed into three or four cross-sectional blocks, fixed in neutral buffered formal saline and embedded in paraffin. Sections were cut at three levels in each block and stained with periodic-acid-Schiff reagent to demonstrate the presence of fungi.

\section{RESULTS}

\section{Haematology}

The haematological results of the untreated animals and of the candida-infected normal animals with and without tetracycline treatment were all within normal limits. The anaemic animals had a mean haemoglobin concentration of $5.18 \mathrm{~g} / \mathrm{dl}$ (range $2 \cdot 3-10 \cdot 3$ ), a mean serum iron of $14 \mu \mathrm{mol} / \mathrm{L}$ (range 6-30), and a mean total iron binding capacity of $120.8 \mu \mathrm{mol} / \mathrm{L}$ (range $49 \cdot 1-167.9$ ) giving a mean transferrin saturation of $14.9 \%$ (range $4 \cdot 3-51 \cdot 6 \%$ ).

\section{Infection with C. albicans}

Normal untreated animals. No evidence of candidal infection or carriage was found either microbiologically or histologically.

Normal animals treated with C. albicans. C. albicans was first recovered from the mouth of one of this group at the start of the third week. The number of animals harbouring $C$. albicans rose to five $(63 \%)$ at the time of the last dose, but fell to zero 1 week later when they were killed. Candida was demonstrated in histological sections from four animals. One animal had lingual and palatal infestation, one had lingual and cheek infestation and two had fungal invasion of the cheek epithelium.

Anaemic animals treated with $C$. albicans. One animal died in this group and has been excluded from the analysis. Anaemic animals gave similar results to the corresponding normal rats. C. albicans was first isolated at the beginning of the third week from one of the nine animals and the number of infected animals rose to six $(67 \%)$ by the last dose. However, $C$. albicans was still recovered from four animals when they were killed. Histopathologically, four animals were shown to have candida invading the oral epithelium.

Comparison of the results obtained with normal and anaemic rats (Fisher exact probability test; Siegel, 1956) showed that the incidence of induced candidal infection and infestation of tongue mucosa did not differ significantly between the groups. However, when the animals were killed, $C$. albicans could not be recovered from any of the normal animals whereas four of the nine anaemic animals remained infected. Although this difference is not statistically significant it may indicate a reduced capacity of anaemic animals to recover from infection.

Normal animals given tetracycline and treated with $C$. albicans. C. albicans was isolated from three of the 20 animals at the beginning of the second week and the number rose to $10(50 \%)$ animals by the time of the last dose. The incidence of recoverable candida did not differ significantly between the groups of rats killed at different times and $C$. albicans was grown from a total of seven of the 19 animals. 
TABLE I

Experimental groups of rats

\begin{tabular}{ccccc}
\hline Group & $\begin{array}{c}\text { Number } \\
\text { of rats }\end{array}$ & $\begin{array}{c}\text { Haematological } \\
\text { state }\end{array}$ & $\begin{array}{c}\text { C.albicans } \\
\text { administration }\end{array}$ & $\begin{array}{c}\text { Tetracycline } \\
\text { treatment }\end{array}$ \\
\hline A & 5 & Normal & - & - \\
B & 8 & Normal & + & - \\
C & 20 & Normal & + & + \\
D & 9 & Anaemic & + & - \\
E & 20 & Anaemic & + & + \\
\hline
\end{tabular}

Histologically, specimens from eight animals showed fungal invasion of oral epithelium.

Anaemic animals given tetracycline and treated with C. albicans. C. albicans was recovered from two of the 20 animals at the beginning of the second week and at the time of the last dose the proportion of animals with recoverable candida had risen to $65 \%$. These animals were killed in three groups at weekly intervals and the incidence of recoverable candida did not differ between these three groups. In all, 16 of the 20 rats remained infected when they were killed. Specimens from 18 animals showed histological evidence of candidal invasion of oral epithelium. Comparison of the normal and anaemic animals given $C$. albicans and tetracycline revealed no significant

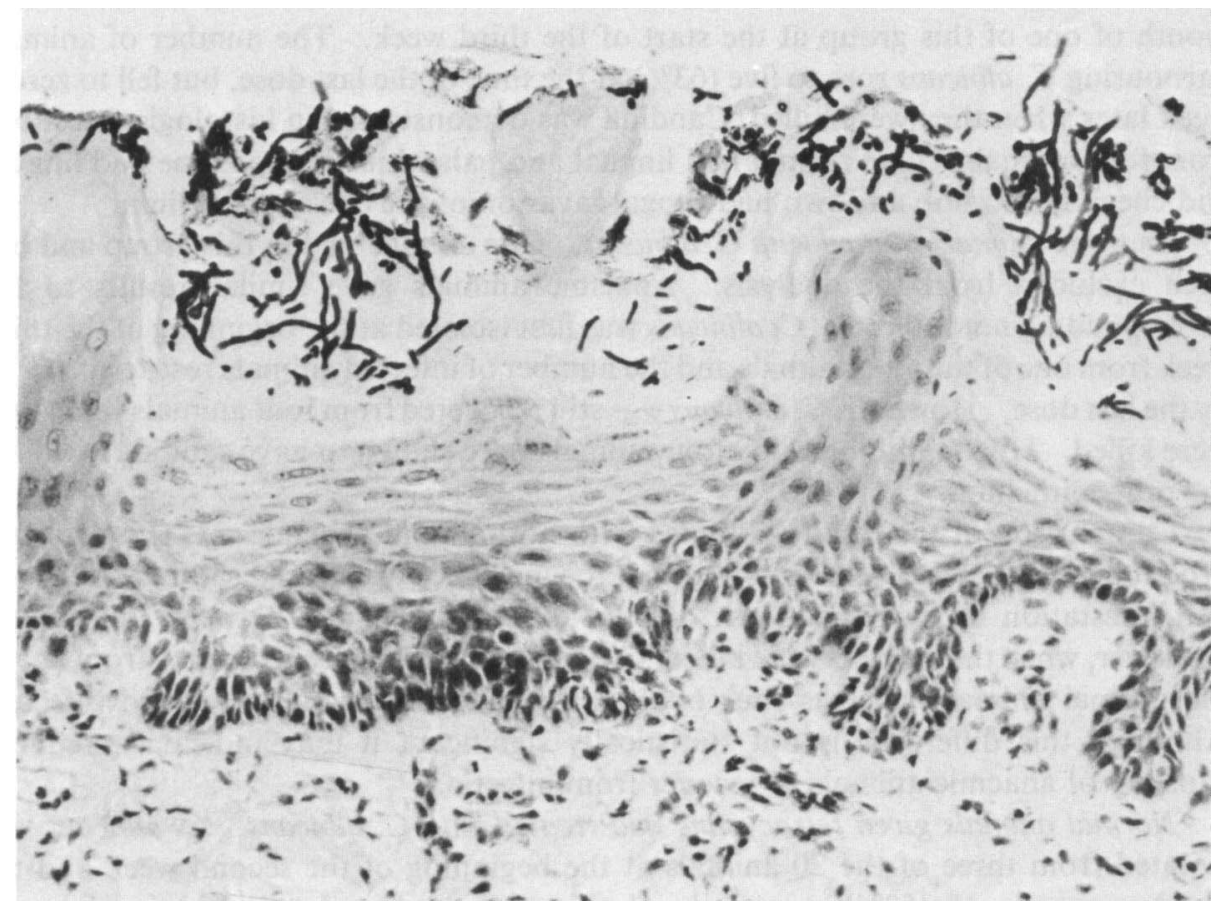

Fig. 1.-Heavy fungal invasion of the keratin of the dorsal surface of the tongue of the rat showing hyphae and blastospores but with only a mild scattered chronic inflammatory cell infiltrate $(\times 80$; PAS diastase). 
difference in the incidence of induced candidal infection up to the time of the last dose. However, as with the first group of animals, the frequency of persistent infection in the anaemic animals when they were killed was higher-16 of 20 anaemic animals and seven of 19 in the non-anaemic group. This difference is significant $(p<0.01$, Chi squared $=7 \cdot 5$ ). Examination of the histological sections stained for fungi showed that significantly more anaemic rats (eight of 19) had candidal infestation of the oral epithelium than normal rats (two of $20 ; p<0.01$, Chi squared $=10.06$ ).

The results were then analysed for the smaller numbers of animals killed at each time and for each intra-oral site examined. For this analysis, the Fisher exact probability test was used (Siegel, 1956).

One week after the last dose of $C$. albicans there was no significant difference in candidal infestation between normal and anaemic rats at any of the intra-oral sites examined. One week later the anaemic group had significantly more animals with infestation of both palate $(p<0.05)$ and cheek epithelium $(p<0.02)$ than normal animals. When the last group was killed, three weeks after the final dose of C. albicans, the anaemic group had significantly more animals with cheek infestation $(p<0.05)$ than the normal rats. There was no difference in the infestation at the other intra-oral sites examined.

Histological examination showed hyphae and blastospores within the keratin layer of the epithelium. In certain sites (e.g., dorsal tongue) growth was particularly heavy (figure) but no subjective difference in fungal load was noted between normal and anaemic animals although animals given tetracycline had heavier fungal infestation. Surprisingly, even at sites where fungal growth was heavy there was little or no inflammatory reaction (figure) and this observation held true for all animal groups.

\section{DisCussion}

The results of this study indicate that iron deficiency does not predispose to oral candidosis in rats. However, if infection becomes established then iron deficient animals are less able to eliminate $C$. albicans from their tissues.

Higgs and Wells (1972) reported that patients with chronic mucocutaneous candidosis and iron deficiency had skin lesions that responded to iron therapy and that the severity of skin involvement paralleled the degree of iron deficiency. In 31 patients with chronic mucocutaneous candidosis, 23 showed evidence of iron deficiency and iron therapy alone produced significant improvement in nine out of 11 of these patients. They postulated that iron deficiency may result in persistent infection which is difficult to eradicate so long as the deficiency remains, a view supported by the present investigation. They also suggested that iron deficiency might produce an epithelial abnormality that is important in the pathogenesis of the infection. Abnormalities are present in iron deficiency anaemia in both man and animals (Rennie and MacDonald, 1982; Rennie, MacDonald and Dagg, 1982). These abnormalities include epithelial atrophy and, in animals, increased thickness of the keratin layer which may provide a more suitable environment for candidal growth, but it is difficult to understand how the epithelial abnormalities allow persistence of infection in iron-deficient animals. Alterations in the immune response that have been described in iron deficiency could result in an inadequate host response to the fungus. Impaired 
lymphocyte transformation and a reduced delayed hypersensitivity reaction have been described in iron deficiency (Joynson et al., 1972) and such defects could result in persistence of infection.

Recently an attempt has been made to induce oral candidosis in anaemic mice (Sofaer, Holbrook and Southam, 1982) and microbiological results similar to those demonstrated in our experiment were found. However the use of a genetic abnormality of iron metabolism is not ideal, as few genetic abnormalities of iron metabolism present in clinical practice and such abnormalities form a very small proportion of the cases with iron deficiency. By far the most common cause of iron deficiency is chronic blood loss or dietary deficiency or both and the rat model is, in this respect, a better model than the mouse in relation to human disease. Moreover, although it is widely known that patients on broad spectrum antibiotics are susceptible to candidal infection the SLA mice given $C$. albicans and antibiotic did not have demonstrable fungal invasion of their epithelium (Sofaer et al., 1982). Further, quantitative analysis of the epithelium of SLA mice has not revealed consistent epithelial abnormalities with, apparently, only the posterior dorsum of the tongue affected.

In a clinical situation it is unlikely that a single nutrient deficiency is the only factor responsible for chronic oral candidal infection. However, the results of the present study indicate that iron deficiency appears to be important in persistent fungal infection. This association has been noticed previously (Jenkins et al., 1977) and it is suggested that patients with chronic oral candidal infection should be screened routinely for iron deficiency.

This work was undertaken while J. S. R. was in receipt of a Medical Research Council Junior Research Fellowship.

\section{REFERENCES}

AMINE, E. K. AND HeGSTED, D. M. 1971. Effect of diet on iron absorption in iron deficient rats. Journal of Nutrition, 101, 927-935.

BlaCK, P. H., KUNZ, L. J. AND SwarTZ, M. N. 1960. Salmonellosis-a review of some unusual aspects. New England Journal of Medicine, 262, 811-817, 864-870, 921-927.

Bullen, J. J., Ward, C. G. AND Wallis, S. N. 1974. Virulence and the role of iron in Pseudomonas aeruginosa infection. Infection and Immunity, 10, 443-450.

Caroline, L., Rosner, F. and Kozinn, P. J. 1969. Elevated serum iron, low unbound transferrin and candidiasis in acute leukemia. Blood, 34, 441-451.

Cawson, R. A. 1963. Denture sore mouth and angular cheilitis. British Dental Journal, 15, 441-449.

Chandra, R. K. 1973. Reduced bactericidal capacity of polymorphs in iron deficiency. Archives of Disease of Childhood, 48, 864-866.

ElIN, R. J. AND WolfF, S. M. 1974. The role of iron in nonspecific resistance to infection induced by endotoxin. Journal of Immunology, 1, 737-745.

FletCher, J., MATHER, J., LeWIS, M. J. AND Whiting, G. 1975. Mouth lesions in iron-deficient anemia: Relationship to Candida albicans in saliva and to impairment of lymphocyte transformation. The Journal of Infectious Diseases, 131, 44-50.

HigGS, J. M. AND Wells, R. S. 1972. Chronic mucocutaneous candidiasis: Associated abnormalities of iron metabolism. British Journal of Dermatology, 86, 88-101.

Jenkins, W. M. M., MacFarlane, T. W., Ferguson, M. M. and Mason, D. K. 1977. Nutritional deficiency in oral candidosis. International Journal of Oral Surgery, 6, 204-210.

Joynson, D. H. M., Jacobs, A., Walker, D. M. AND Dolby, A. E. 1972. Defect of 
cell-mediated immunity in patients with iron deficiency anaemia. Lancet, 2, 1058-1059.

LODDER, J. 1970. The yeasts. A taxonomic study, 2nd Ed. North Holland, Amsterdam, p. 1384.

McCall, M. G., Newman, G. E., O'Brien, J. R. P., Valberg, L. S. and Witts, L. J. 1962. Studies in iron metabolism. 1. The experimental production of iron deficiency in the growing rat. British Journal of Nutrition, 16, 297-304.

MACKENZIE, D. W. R. 1962. Serum tube identification of Candida albicans. Journal of Clinical Pathology, 15, 563-565.

Masawe, A. E. J., Muindi, J. M. and SwaI, G. B. R. 1974. Infections in iron deficiency and other types of anaemia in the tropics. Lancet, 2, 314-317.

RenNiE, J. S. AND MacDonald, D. G. 1982. Quantitative histological analysis of the epithelium of the ventral surface of hamster tongue in experimental iron deficiency. Archives of Oral Biology, 27, 393-397.

ReNnie, J. S., MacDonald, D. G. AND DAGG, J. H. 1982. Quantitative analysis of human buccal epithelium in iron deficiency anaemia. Journal of Oral Pathology, 11, 39-46.

RingelhanN, B., Konotey-Ahulu, F. AND Dodu, S. R. A. 1970. Studies on iron metabolism in sickle cell anaemia, sickle cell haemoglobin $C$ disease, and haemoglobin $C$ disease, using a large volume liquid scintillation counter. Journal of Clinical Pathology, 23, 127-134.

RusSELL, C. AND JONES, J. H. 1973. Effects of oral inoculation of Candida albicans in tetracycline-treated rats. Journal of Medical Microbiology, 6, 275-279.

SChADE, A. L. AND CAROLINE, L. 1944. Raw hen egg white and the role of iron in growth inhibition of Shigella dysenteriae, Staphylococcus aureus, Escherichia coli and Saccharomyces cerevisiae. Science, 100, 14-15.

Schade, A. L. AND CAROLINE, L. 1946. An iron-binding component in human blood plasma. Science, 104, 340-341.

SIEGEL, S. 1956. Non-parametric Statistics for the Behavioral Sciences. McGraw-Hill Book Company Inc., New York.

Sofaer, J. A., HolbrooK, W. P. AND Southam, J. C. 1982. Experimental oral infection with the yeast Candida albicans in mice with or without inherited iron-deficiency anaemia (SLA). Archives of Oral Biology, 27, 497-503. 